KYUNGPOOK Math. J. 50(2010), 483-497

\title{
Dynamics of Recursive Sequence of Order Two
}

Elsayed Mohammed Elsayed

King AbdulAziz University, Faculty of Science, Mathematics Department, P. O. Box 80203, Jeddah 21589, Saudi Arabia;

Department of Mathematics, Faculty of Science, Mansoura University, Mansoura 35516, Egypt

e-mail : emelsayed@mans.edu.eg; emmelsayed@yahoo.com

ABSTRACT. In this paper we study some qualitative behavior of the solutions of the difference equation

$$
x_{n+1}=a x_{n}+\frac{b x_{n}}{c x_{n}-d x_{n-1}}, \quad n=0,1, \ldots,
$$

where the initial conditions $x_{-1}, x_{0}$ are arbitrary real numbers and $a, b, c, d$ are positive constants with $c x_{0}-d x_{-1} \neq 0$.

\section{Introduction}

In this paper we deal with some properties of the solutions of the difference equation

$$
x_{n+1}=a x_{n}+\frac{b x_{n}}{c x_{n}-d x_{n-1}}, \quad n=0,1, \ldots
$$

where the initial conditions $x_{-1}, x_{0}$ are arbitrary real numbers and $a, b, c, d$ are positive constants with $c x_{0}-d x_{-1} \neq 0$.

Recently there has been a lot of interest in studying the global attractivity, boundedness character and the periodic nature of nonlinear difference equations. For some results in this area, see for example [21-41].

Many researchers have investigated the behavior of the solution of difference equations for example:

In [5] Elabbasy et al. investigated the global stability character, boundedness and the periodicity of solutions of the difference equation

$$
x_{n+1}=\frac{\alpha x_{n}+\beta x_{n-1}+\gamma x_{n-2}}{A x_{n}+B x_{n-1}+C x_{n-2}} .
$$

Elabbasy et al. [6] investigated the global stability, boundedness, periodicity char-

Received April 4, 2010; accepted December 8, 2010.

2000 Mathematics Subject Classification: 39A10.

Key words and phrases: difference equations, stability, periodicity, solution of the difference equation. 
acter and gave the solution of some special cases of the difference equation

$$
x_{n+1}=\frac{\alpha x_{n-k}}{\beta+\gamma \prod_{i=0}^{k} x_{n-i}} .
$$

Elabbasy et al. [7] investigated the global stability, periodicity character and gave the solution of some special cases of the difference equation

$$
x_{n+1}=\frac{d x_{n-l} x_{n-k}}{c x_{n-s}-b}+a .
$$

Aloqeili [1] obtained the form of the solutions of the difference equation

$$
x_{n+1}=\frac{x_{n-1}}{a-x_{n} x_{n-1}} .
$$

Simsek et al. [32] obtained the solution of the difference equation

$$
x_{n+1}=\frac{x_{n-3}}{1+x_{n-1}} .
$$

Other related results on rational difference equations can be found in refs. [2-20].

Here, we recall some notations and results which will be useful in our investigation.

Let $I$ be some interval of real numbers and the function $f$ has continuous partial derivatives on $I^{k+1}$ where $I^{k+1}=I \times I \times \ldots \times I \quad(k+1-$ times $)$. Then, for initial conditions $x_{-k}, x_{-k+1}, \ldots, x_{0} \in I$, it is easy to see that the difference equation

$$
x_{n+1}=f\left(x_{n}, x_{n-1}, \ldots, x_{n-k}\right), \quad n=0,1, \ldots,
$$

has a unique solution $\left\{x_{n}\right\}_{n=-k}^{\infty}$.

A point $\bar{x} \in I$ is called an equilibrium point of Eq.(2) if

$$
\bar{x}=f(\bar{x}, \bar{x}, \ldots, \bar{x}) .
$$

That is, $x_{n}=\bar{x}$ for $n \geq 0$, is a solution of Eq.(2), or equivalently, $\bar{x}$ is a fixed point of $f$.

Definition 1(Stability).

(i) The equilibrium point $\bar{x}$ of Eq.(2) is locally stable if for every $\epsilon>0$, there exists $\delta>0$ such that for all $x_{-k}, x_{-k+1}, \ldots, x_{-1}, x_{0} \in I$ with

$$
\left|x_{-k}-\bar{x}\right|+\left|x_{-k+1}-\bar{x}\right|+\ldots+\left|x_{0}-\bar{x}\right|<\delta,
$$

we have

$$
\left|x_{n}-\bar{x}\right|<\epsilon \quad \text { for all } n \geq-k .
$$

(ii) The equilibrium point $\bar{x}$ of Eq.(2) is locally asymptotically stable if $\bar{x}$ is locally stable solution of Eq.(2) and there exists $\gamma>0$, such that for all $x_{-k}, x_{-k+1}, \ldots, x_{-1}, x_{0} \in I$ with

$$
\left|x_{-k}-\bar{x}\right|+\left|x_{-k+1}-\bar{x}\right|+\ldots+\left|x_{0}-\bar{x}\right|<\gamma,
$$


we have

$$
\lim _{n \rightarrow \infty} x_{n}=\bar{x} .
$$

(iii) The equilibrium point $\bar{x}$ of Eq.(2) is global attractor if for all $x_{-k}, x_{-k+1}, \ldots, x_{-1}, x_{0} \in$ $I$, we have

$$
\lim _{n \rightarrow \infty} x_{n}=\bar{x} .
$$

(iv) The equilibrium point $\bar{x}$ of Eq.(2) is globally asymptotically stable if $\bar{x}$ is locally stable, and $\bar{x}$ is also a global attractor of Eq.(2).

(v) The equilibrium point $\bar{x}$ of Eq.(2) is unstable if $\bar{x}$ is not locally stable.

The linearized equation of Eq.(2) about the equilibrium $\bar{x}$ is the linear difference equation

$$
y_{n+1}=\sum_{i=0}^{k} \frac{\partial f(\bar{x}, \bar{x}, \ldots, \bar{x})}{\partial x_{n-i}} y_{n-i}
$$

Now assume that the characteristic equation associated with Eq.(3) is

$$
p(\lambda)=p_{0} \lambda^{k}+p_{1} \lambda^{k-1}+\ldots+p_{k-1} \lambda+p_{k}=0
$$

where $p_{i}=\frac{\partial f(\bar{x}, \bar{x}, \ldots, \bar{x})}{\partial x_{n-i}}$.

Theorem A([28]). Assume that $p_{i} \in R, i=1,2, \ldots$ and $k \in\{0,1,2, \ldots\}$. Then

$$
\sum_{i=1}^{k}\left|p_{i}\right|<1
$$

is a sufficient condition for the asymptotic stability of the difference equation

$$
y_{n+k}+p_{1} y_{n+k-1}+\ldots+p_{k} y_{n}=0, \quad n=0,1, \ldots .
$$

Consider the following equation

$$
x_{n+1}=f\left(x_{n}, x_{n-1}\right) .
$$

The following theorems will be useful for the proof of our main results in this paper.

Theorem B([27]). Let $f:[a, b]^{2} \rightarrow[a, b]$ be a continuous function, where $a$ and $b$ are real numbers with $a<b$. Suppose that $f$ satisfies the following conditions:

(a) $f(x, y)$ is non-decreasing in $x \in[a, b]$ for each fixed $y \in[a, b]$, and is nondecreasing in $y \in[a, b]$ for each $x \in[a, b]$.

(b) If $(m, M)$ is a solution of the system

$$
m=f(m, m) \quad \text { and } \quad M=f(M, M),
$$


then

$$
m=M \text {. }
$$

Then there exists exactly one equilibrium $\bar{x}$ of Eq. (6), and every solution of Eq.(6) converges to $\bar{x}$.

Theorem $\mathbf{C}([\mathbf{2 7}])$. Let $f:[a, b]^{2} \rightarrow[a, b]$ be a continuous function, where $a$ and $b$ are real numbers with $a<b$. Suppose that $f$ satisfies the following conditions:

(a) $f(x, y)$ is non-increasing in $x \in[a, b]$ for each fixed $y \in[a, b]$, and is nondecreasing in $y \in[a, b]$ for each $x \in[a, b]$.

(b) If $(m, M)$ is a solution of the system

$$
m=f(M, m) \quad \text { and } \quad M=f(m, M),
$$

then

$$
m=M \text {. }
$$

Then there exists exactly one equilibrium $\bar{x}$ of Eq. (6), and every solution of Eq.(6) converges to $\bar{x}$.

\section{Periodic solutions}

In this section we study the existence of periodic solutions of Eq.(1). The following theorem states the necessary and sufficient conditions that this equation has periodic solutions.

Theorem 1. Eq.(1) has positive prime period two solutions if and only if

$$
(c+d)(a+1)>4 d, \quad d \neq a c .
$$

Proof. First suppose that there exists a prime period two solution

$$
\ldots, p, q, p, q, \ldots,
$$

of Eq.(1). We will prove that Condition (7) holds.

We see from Eq.(1) that

$$
p=a q+\frac{b q}{c q-d p},
$$

and

$$
q=a p+\frac{b p}{c p-d q} .
$$

Then

$$
c p q-d p^{2}=a c q^{2}-a d p q+b q,
$$


and

$$
c p q-d q^{2}=a c p^{2}-a d p q+b p .
$$

Subtracting (9) from (8) gives

$$
d\left(q^{2}-p^{2}\right)=a c\left(q^{2}-p^{2}\right)+b(q-p) .
$$

Since $p \neq q$, it follows that

$$
p+q=\frac{b}{d-a c} .
$$

Again, adding (8) and (9) yields

$$
2 c p q-d\left(p^{2}+q^{2}\right)=a c\left(p^{2}+q^{2}\right)-2 a d p q+b(p+q) .
$$

It follows by (10), (11) and the relation

$$
p^{2}+q^{2}=(p+q)^{2}-2 p q \text { for all } p, q \in R,
$$

that

$$
p q=\frac{b^{2} d}{(d-a c)^{2}(c+d)(a+1)} .
$$

Now it is clear from Eq.(10) and Eq.(12) that $p$ and $q$ are the two positive distinct roots of the quadratic equation

$$
(d-a c) t^{2}-b t+\frac{b^{2} d}{(d-a c)(c+d)(a+1)}=0 .
$$

and so

$$
b^{2}>\frac{4 b^{2} d}{(c+d)(a+1)} .
$$

Therefore inequality (7) holds.

Second suppose that inequality (7) is true. We will show that Eq.(1) has a prime period two solution.

Assume that

$$
p=\frac{b+\alpha}{2(d-a c)}
$$

and

$$
q=\frac{b-\alpha}{2(d-a c)},
$$

where $\alpha=\sqrt{b^{2}-\frac{4 b^{2} d}{(c+d)(a+1)}}$. 
From inequality (7) it follows that $\alpha$ is a real positive numbers, therefore $p$ and $q$ are distinct positive real numbers.

Set

$$
x_{-1}=p \text { and } x_{0}=q .
$$

We show that $x_{1}=x_{-1}=p \quad$ and $\quad x_{2}=x_{0}=q$.

It follows from Eq.(1) that

$$
\begin{gathered}
x_{1}=a q+\frac{b q}{c q-d p}=\frac{a c q^{2}-a d p q+b q}{c q-d p} \\
=\frac{a c\left[\frac{b-\alpha}{2(d-a c)}\right]^{2}-a d\left[\frac{b^{2} d}{(d-a c)^{2}(c+d)(a+1)}\right]+b\left[\frac{b-\alpha}{2(d-a c)}\right]}{c\left[\frac{b-\alpha}{2(d-a c)}\right]-d\left[\frac{b+\alpha}{2(d-a c)}\right]} .
\end{gathered}
$$

Multiplying the denominator and numerator by $4(d-a c)^{2}$

$$
x_{1}=\frac{2 b^{2} d-\frac{4 a b^{2} c d+4 a b^{2} d^{2}}{(c+d)(a+1)}-2 b d \alpha}{2(d-a c)\{c b-b d-(c+d) \alpha\}} .
$$

Multiplying the denominator and numerator by $\{c b-b d+(c+d) \alpha\}\{(c+d)(a+1)\}$ we get

$x_{1}=\frac{\left[\left(4 b^{3} d^{3}+4 b^{3} c d^{2}-4 a b^{3} c^{2} d-4 a b^{3} c d^{2}\right)+\left(4 b^{2} c d^{2}+4 b^{2} d^{3}-4 a b^{2} c^{2} d-4 a b^{2} c d^{2}\right) \alpha\right]}{2(d-a c)\left\{4 b^{2} c d^{2}+4 b^{2} d^{3}-4 a b^{2} c^{2} d-4 a b^{2} c d^{2}\right\}}$.

Dividing the denominator and numerator by $\left\{4 b^{2} c d^{2}+4 b^{2} d^{3}-4 a b^{2} c^{2} d-4 a b^{2} c d^{2}\right\}$ gives

$$
x_{1}=\frac{b+\alpha}{2(d-a c)}=p .
$$

Similarly as before one can easily show that

$$
x_{2}=q .
$$

Then it follows by induction that

$$
x_{2 n}=q \quad \text { and } \quad x_{2 n+1}=p \quad \text { for all } \quad n \geq-1 .
$$

Thus Eq.(1) has the positive prime period two solution

$$
\ldots, p, q, p, q, \ldots,
$$

where $p$ and $q$ are the distinct roots of the quadratic equation (13) and the proof is complete. 


\section{Local stability of the equilibrium point}

In this section we study the local stability character of the solutions of Eq.(1). The equilibrium points of Eq.(1) are given by the relation

$$
\bar{x}=a \bar{x}+\frac{b \bar{x}}{c \bar{x}-d \bar{x}} .
$$

If $(c-d)(1-a)>0$, then the only positive equilibrium point of Eq.(1) is given by

$$
\bar{x}=\frac{b}{(c-d)(1-a)} .
$$

Let $g:(0, \infty)^{2} \longrightarrow(0, \infty)$ be a function defined by

$$
g(u, v)=a u+\frac{b u}{c u-d v}
$$

Therefore

$$
\begin{aligned}
& \frac{\partial g(u, v)}{\partial u}=a-\frac{b d v}{(c u-d v)^{2}} \\
& \frac{\partial g(u, v)}{\partial v}=\frac{b d u}{(c u-d v)^{2}}
\end{aligned}
$$

Then we see that

$$
\begin{aligned}
\frac{\partial g(\bar{x}, \bar{x})}{\partial u} & =a-\frac{d(1-a)}{(c-d)}=p_{0}, \\
\frac{\partial g(\bar{x}, \bar{x})}{\partial v} & =\frac{d(1-a)}{(c-d)}=p_{1} .
\end{aligned}
$$

Then the linearized equation of Eq.(1) about $\bar{x}$ is

$$
y_{n+1}-p_{0} y_{n-1}-p_{1} y_{n}=0 .
$$

Theorem 2. Assume that

$$
|a c-d|+d|1-a|<|c-d| .
$$

Then the equilibrium point of Eq.(1) is locally asymptotically stable.

Proof. It is follows by Theorem A that, Eq.(1) is asymptotically stable if

$$
\left|p_{1}\right|+\left|p_{0}\right|<1
$$

That is

$$
\left|a-\frac{d(1-a)}{(c-d)}\right|+\left|\frac{d(1-a)}{(c-d)}\right|<1,
$$


then

$$
|a(c-d)-d(1-a)|+|d(1-a)|<|c-d| .
$$

Thus

$$
|a c-d|+d|1-a|<|c-d|
$$

The proof is complete.

\section{Global attractor of the equilibrium point of Eq.(1)}

In this section we investigate the global attractivity character of solutions of Eq.(1).

Theorem 3. The equilibrium point $\bar{x}$ of Eq.(1) is a global attractor if ac $>d$.

Proof. Let $p, q$ are a real numbers and assume that $g:[p, q]^{2} \longrightarrow[p, q]$ be a function defined by Eq.(14).Therefore

$$
\begin{aligned}
& \frac{\partial g(u, v)}{\partial u}=a-\frac{b d v}{(c u-d v)^{2}}, \\
& \frac{\partial g(u, v)}{\partial v}=\frac{b d u}{(c u-d v)^{2}} .
\end{aligned}
$$

Case (1) If $a-\frac{b d v}{(c u-d v)^{2}}>0$, then we can easily see that the function $g(u, v)$ increasing in $u, v$.

Suppose that $(m, M)$ is a solution of the system

$$
m=g(m, m) \quad \text { and } \quad M=g(M, M) .
$$

Then from Eq.(1), we see that

$$
m=a m+\frac{b m}{c m-d m}, \quad M=a M+\frac{b M}{c M-d M},
$$

then

$$
(M-m)=a(M-m), \quad a \neq 1 .
$$

Thus

$$
M=m .
$$

It follows by the Theorem B that $\bar{x}$ is a global attractor of Eq.(1).

Case (2) If $a-\frac{b d v}{(c u-d v)^{2}}<0$, then we can easily see that the function $g(u, v)$ decreasing in $u$ and increasing in $v$.

Suppose that $(m, M)$ is a solution of the system

$$
M=g(m, M) \quad \text { and } \quad m=g(M, m) .
$$


Then from Eq.(1), we see that

$$
\begin{gathered}
m=a M+\frac{b M}{c M-d m}, \quad M=a m+\frac{b m}{c m-d M}, \\
c M m-a c M^{2}-d m^{2}+a d M m=b M, \quad c M m-a c m^{2}-d M^{2}+a d M m=b m,
\end{gathered}
$$

then

$$
\left(M^{2}-m^{2}\right)(d-a c)=b(M-m), \quad a c>d .
$$

Thus

$$
M=m .
$$

It follows by the Theorem $\mathrm{C}$ that $\bar{x}$ is a global attractor of Eq.(1) and then the proof is complete.

\section{Special case of Eq.(1)}

In this section we study the following special case of Eq.(1)

$$
x_{n+1}=x_{n}+\frac{x_{n}}{x_{n}-x_{n-1}},
$$

where the initial conditions $x_{-1}, x_{0}$ are arbitrary real numbers with $x_{-1}, x_{0} \in$ $R /\{0\}$ and $x_{-1} \neq x_{0}$.

\subsection{The solution form of Eq.(16)}

In this section we give a specific form of the solutions of Eq.(16).

Ttheorem 4. Let $\left\{x_{n}\right\}_{n=-1}^{\infty}$ be the solution of Eq.(16) satisfying $x_{-1}=k, x_{0}=$ $h$, with $k \neq h, k, h \in R /\{0\}$. Then for $n=0,1, \ldots$

$$
\begin{aligned}
x_{2 n-1} & =k+n\left(h-k+(n-1)+\frac{h}{h-k}\right), \\
x_{2 n} & =h+n\left(h-k+n+\frac{h}{h-k}\right) .
\end{aligned}
$$

Proof. For $n=0$ the result holds. Now suppose that $n>0$ and that our assumption holds for $n-1$. That is;

$$
\begin{aligned}
& x_{2 n-3}=k+(n-1)\left(h-k+(n-2)+\frac{h}{h-k}\right), \\
& x_{2 n-2}=h+(n-1)\left(h-k+(n-1)+\frac{h}{h-k}\right) .
\end{aligned}
$$

Now, it follows from Eq.(16) that

$$
x_{2 n-1}=x_{2 n-2}+\frac{x_{2 n-2}}{x_{2 n-2}-x_{2 n-3}}=h+(n-1)\left(h-k+(n-1)+\frac{h}{h-k}\right)
$$




$$
\begin{gathered}
+\frac{h+(n-1)\left(h-k+(n-1)+\frac{h}{h-k}\right)}{\left(h+(n-1)\left(h-k+(n-1)+\frac{h}{h-k}\right)\right)-\left(k+(n-1)\left(h-k+(n-2)+\frac{h}{h-k}\right)\right)} \\
=h+(n-1)\left(h-k+(n-1)+\frac{h}{h-k}\right)+\frac{h+(n-1)\left(h-k+(n-1)+\frac{h}{h-k}\right)}{h-k+(n-1)} .
\end{gathered}
$$

Multiplying the denominator and numerator by $(h-k)$ we get

$$
\begin{aligned}
x_{2 n-1} & =k+(n-1)\left(h-k+(n-1)+\frac{h}{h-k}\right)+\frac{(h+(n-1)(h-k))}{(h-k)}+(h-k) \\
& =k+(n-1)\left(h-k+(n-1)+\frac{h}{h-k}\right)+(h-k)+(n-1)+\frac{h}{(h-k)},
\end{aligned}
$$

then we have

$$
x_{2 n-1}=k+n\left(h-k+(n-1)+\frac{h}{h-k}\right) .
$$

Also, we get from Eq.(16)

$$
\begin{aligned}
x_{2 n} & =x_{2 n-1}+\frac{x_{2 n-1}}{x_{2 n-1}-x_{2 n-2}} \\
& =k+n\left(h-k+(n-1)+\frac{h}{h-k}\right)+\frac{k+n\left(h-k+(n-1)+\frac{h}{h-k}\right)}{(n-1)+\frac{h}{(h-k)}} .
\end{aligned}
$$

Multiplying the denominator and numerator by $(h-k)$ we get

$$
\begin{aligned}
x_{2 n}= & k+n\left(h-k+(n-1)+\frac{h}{h-k}\right) \\
& +\frac{\left(k(h-k)+n(h-k)^{2}\right)+(n(n-1)(h-k)+n h)}{(n-1)(h-k)+h},
\end{aligned}
$$

or

$$
x_{2 n}=k+n\left(h-k+(n-1)+\frac{h}{h-k}\right)+(h-k+n) .
$$

Thus we obtain

$$
x_{2 n}=h+n\left(h-k+n+\frac{h}{h-k}\right) .
$$

Hence, the proof is completed.

Remark 2. It is easy to see that every solution of Eq.(16) is unbounded. 


\section{Numerical examples}

Here we consider numerical examples which represent different types of solutions to Eq. (1).

Example 1. Consider $x_{-1}=29, x_{0}=8$ and $a=b=c=d=1$. See Fig. 1.

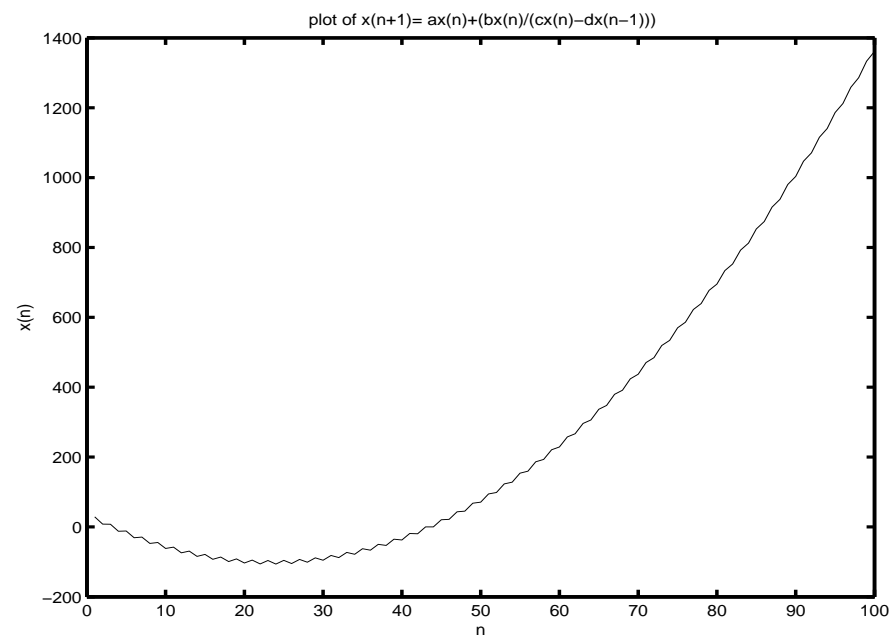

Fig. 1

Example 2. See Fig. 2, since $x_{-1}=9, x_{0}=6$ and $a=0.8, b=3, c=5, d=2$.

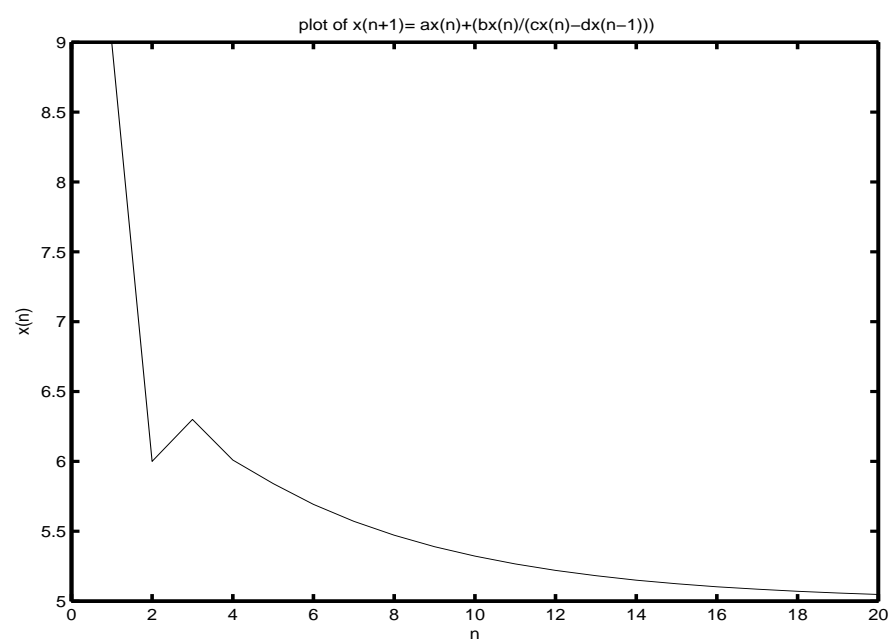

Fig. 2 
Example 3. See Fig. 3, since $x_{-1}=7, x_{0}=9$ and $a=0.5, b=13, c=6, d=3$.

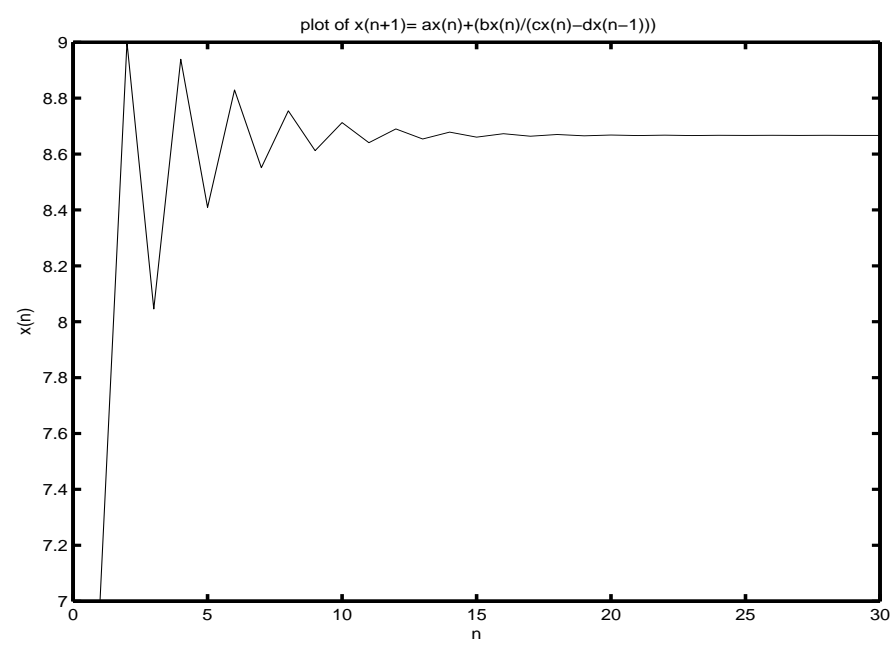

Fig. 3

Example 4. See Fig. 4 , since $x_{-1}=1.928\left(=p=\frac{b+\alpha}{2(d-a c)}\right), x_{0}=1.071$ $\left(=q=\frac{b-\alpha}{2(d-a c)}\right)$ and $a=2, b=6, c=4, d=10$.

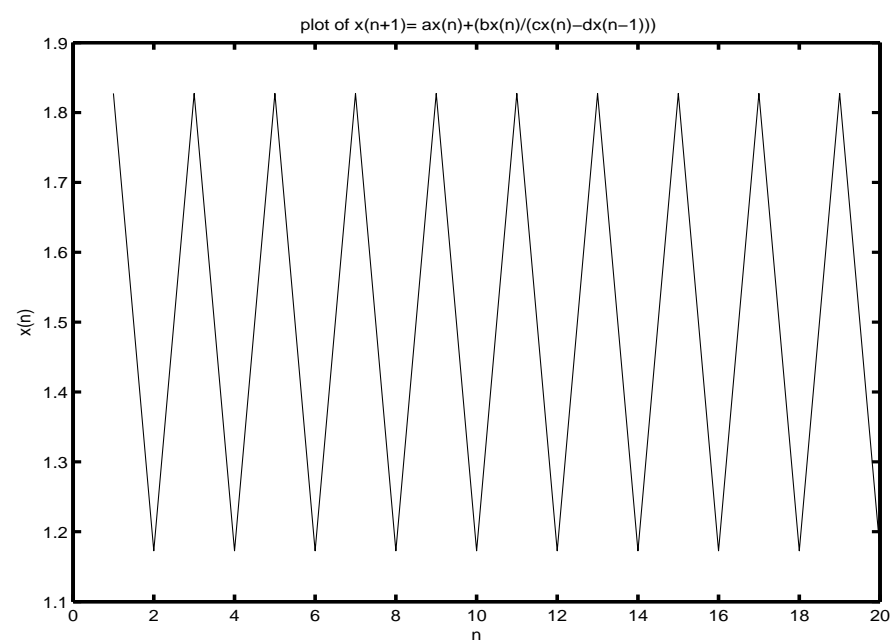

Fig. 4 


\section{References}

[1] M. Aloqeili, Dynamics of a rational difference equation, Appl. Math. Comp., 176(2)(2006), 768-774.

[2] C. Cinar, On the positive solutions of the difference equation $x_{n+1}=\frac{x_{n-1}}{1+x_{n} x_{n-1}}$, Applied Mathematics and Computation, 150(2004), 21-24.

[3] C. Cinar, On the positive solutions of the difference equation $x_{n+1}=\frac{a x_{n-1}}{1+b x_{n} x_{n-1}}$, Applied Mathematics and Computation, 156(2004), 587-590.

[4] C. Cinar, On the difference equation $x_{n+1}=\frac{x_{n-1}}{-1+x_{n} x_{n-1}}$, Applied Mathematics and Computation, 158(2004), 813-816.

[5] E. M. Elabbasy, H. El-Metwally and E. M. Elsayed, Global attractivity and periodic character of a fractional difference equation of order three, Yokohama Math. J., 53(2007), 89-100.

[6] E. M. Elabbasy, H. El-Metwally and E. M. Elsayed, On the difference equation $x_{n+1}=$ $\frac{\alpha x_{n-k}}{\beta+\gamma \prod_{i=0}^{k} x_{n-i}}$, J. Conc. Appl. Math., 5(2)(2007), 101-113.

[7] E. M. Elabbasy, H. El-Metwally and E. M. Elsayed, Qualitative behavior of higher order difference equation, Soochow Journal of Mathematics, 33(4)(2007), 861-873.

[8] E. M. Elabbasy, H. El-Metwally and E. M. Elsayed, On the difference equation $x_{n+1}=$ $\frac{a_{0} x_{n}+a_{1} x_{n-1}+\ldots+a_{k} x_{n-k}}{b_{0} x_{n}+b_{1} x_{n-1}+\ldots+b_{k} x_{n-k}}$, Mathematica Bohemica, 133(2)(2008), 133-147.

[9] E. M. Elabbasy and E. M. Elsayed, On the global attractivity of difference equation of higher order, Carpathian Journal of Mathematics, 24(2)(2008), 45 - 53.

[10] E. M. Elabbasy and E. M. Elsayed, On the solutions of a class of difference equations of higher order, International Journal of Mathematics and Statistics, 6(A09)(2010), 57-68.

[11] E. M. Elabbasy and E. M. Elsayed, Dynamics of a rational difference equation, Chinese Annals of Mathematics, Series B, 30(2)(2009), 187-198.

[12] H. El-Metwally, E. A. Grove, and G. Ladas, A global convergence result with applications to periodic solutions, J. Math. Anal. Appl., 245(2000), 161-170.

[13] H. El-Metwally, E. A. Grove, G. Ladas and H. D. Voulov, On the global attractivity and the periodic character of some difference equations, J. Differ. Equations Appl., 7(2001), 1-14.

[14] H. El-Metwally, Global behavior of an economic model, Chaos, Solitons and Fractals, 33(2007), 994-1005.

[15] H. El-Metwally and M. M. El-Afifi, On the behavior of some extension forms of some population models, Chaos, Solitons and Fractals, 36(2008), 104-114.

[16] E. M. Elsayed, On the solution of recursive sequence of order two, Fasciculi Mathematici, 40(2008), 5-13.

[17] E. M. Elsayed, Dynamics of a recursive sequence of higher order, Communications on Applied Nonlinear Analysis, 16(2)(2009), 37-50. 
[18] E. M. Elsayed, On the Difference Equation $x_{n+1}=\frac{x_{n-5}}{-1+x_{n-2} x_{n-5}}$, International Journal of Contemporary Mathematical Sciences, 3(33)(2008), 1657-1664.

[19] E. M. Elsayed, Qualitative behavior of difference equation of order three, Acta Scientiarum Mathematicarum (Szeged), 75(1-2), 113-129.

[20] E. M. Elsayed, Qualitative behavior of s rational recursive sequence, Indagationes Mathematicae, New Series, 19(2)(2008), 189-201.

[21] E. M. Elsayed, On the Global attractivity and the solution of recursive sequence, Studia Scientiarum Mathematicarum Hungarica, 47(3)(2010), 401-418.

[22] E. M. Elsayed, Qualitative properties for a fourth order rational difference equation, Acta Applicandae Mathematicae, 110(2)(2010), 589-604.

[23] E. M. Elsayed, Qualitative behavior of difference equation of order two, Mathematical and Computer Modelling, 50(2009), 1130-1141.

[24] E. A. Grove and G. Ladas, Periodicities in Nonlinear Difference Equations, Chapman \& Hall / CRC Press, 2005.

[25] A. E. Hamza, S. G. Barbary, Attractivity of the recursive sequence $x_{n+1}=(\alpha-$ $\left.\beta x_{n}\right) F\left(x_{n-1}, \ldots, x_{n-k}\right)$, Mathematical and Computer Modelling, 48(11-12)(2008), 1744-1749.

[26] T. F. Ibrahim, On the third order rational difference equation $x_{n+1}=$ $\frac{x_{n} x_{n-2}}{x_{n-1}\left(a+b x_{n} x_{n-2}\right)}$, Int. J. Contemp. Math. Sciences, 4(27)(2009), 1321-1334.

[27] V. L. Kocic and G. Ladas, Global Behavior of Nonlinear Difference Equations of Higher Order with Applications, Kluwer Academic Publishers, Dordrecht, 1993.

[28] M. R. S. Kulenovic and G. Ladas, Dynamics of Second Order Rational Difference Equations with Open Problems and Conjectures, Chapman \& Hall / CRC Press, 2001.

[29] A. Rafiq, Convergence of an iterative scheme due to Agarwal et al., Rostock. Math. Kolloq., 61(2006), 95-105.

[30] M. Saleh and S. Abu-Baha, Dynamics of a higher order rational difference equation, Appl. Math. Comp., 181(2006), 84-102.

[31] M. Saleh and M. Aloqeili, On the difference equation $x_{n+1}=A+\frac{x_{n}}{x_{n-k}}$, Appl. Math. Comp., 171(2005), 862-869.

[32] D. Simsek, C. Cinar and I. Yalcinkaya, On the recursive sequence $x_{n+1}=\frac{x_{n-3}}{1+x_{n-1}}$, Int. J. Contemp. Math. Sci., 1(10)(2006), 475-480.

[33] C. Wang, S. Wang and X. Yan, Global asymptotic stability of 3-species mutualism models with diffusion and delay effects, Discrete Dynamics in Natural and Science, Volume 2009, Article ID 317298, 20 pages.

[34] C. Wang, F. Gong, S. Wang, L. LI and Q. Shi, Asymptotic behavior of equilibrium point for a class of nonlinear difference equation, Advances in Difference Equations, Volume 2009, Article ID 214309, 8 pages.

[35] I. Yalçınkaya, C. Cinar and M. Atalay, On the solutions of systems of difference equations, Advances in Difference Equations, Vol. 2008, Article ID 143943, 9 pages, doi: $10.1155 / 2008 / 143943$. 
[36] I. Yalçınkaya, On the global asymptotic stability of a second-order system of difference equations, Discrete Dynamics in Nature and Society, Vol. 2008, Article ID 860152, 12 pages, doi: 10.1155/2008/860152.

[37] I. Yalçınkaya, C. Cinar and M. Atalay, On the solutions of systems of difference equations, Advances in Difference Equations, Vol. 2008, Article ID 143943, 9 pages, doi: $10.1155 / 2008 / 143943$.

[38] I. Yalçınkaya, On the global asymptotic stability of a second-order system of difference equations, Discrete Dynamics in Nature and Society, Vol. 2008, Article ID 860152, 12 pages, doi: 10.1155/2008/860152.

[39] I. Yalçınkaya, On the difference equation $x_{n+1}=\alpha+\frac{x_{n-m}}{x_{n}^{k}}$, Discrete Dynamics in Nature and Society, Vol. 2008, Article ID 805460, 8 pages, doi: 10.1155/2008/ 805460.

[40] E. M. E. Zayed and M. A. El-Moneam, On the rational recursive sequence $x_{n+1}=$ $\frac{\alpha+\beta x_{n}+\gamma x_{n-1}}{A+B x_{n}+C x_{n-1}}$, Communications on Applied Nonlinear Analysis, 12(4)(2005), $15-28$.

[41] E. M. E. Zayed and M. A. El-Moneam, On the rational recursive sequence $x_{n+1}=$ $\frac{\alpha x_{n}+\beta x_{n-1}+\gamma x_{n-2}+\delta x_{n-3}}{A x_{n}+B x_{n-1}+C x_{n-2}+D x_{n-3}}$, Comm. Appl. Nonlinear Analysis, 12(2005), 15-28. 